\title{
Polarimetric Radar Studies of Atmospheric Ice Particles
}

\author{
J. Vivekanandan, V. N. Bringi, M. Hagen, and P. Meischner
}

\begin{abstract}
Single scattering properties of ice crystals are described at microwave frequencies using discrete dipole approximations and Rayleigh scattering techniques. For a given shape, the average bulk densities of ice crystals can be estimated using the ratio of the copolarized radar signal in a linear (horizontal, vertical) polarization basis. Reflectivity depends on the ice content $\left(\mathrm{g} \cdot \mathrm{m}^{-3}\right)$, and also on both size distribution parameters and average bulk density of the scatterers. Differential propagation phase is primarily a function of shape, ice water content, and is independent of size distribution parameters. Thus, by using a combination of polarimetric radar measurements, average ice content, bulk density, and shape of distributed scatterers can be inferred. These techniques become quite complex in the case of a winter storm where scatterers can exist with varying shape and bulk densities. Polarimetric radar properties of such complex distributed scatterers are modeled. Physical variations in the relation among ice water content, reflectivity, and differential propagation phase are considered with respect to change in the shape of size distribution, bulk density, and average shape of the scatterers. Also, simultaneous polarimetric radar observations and in situ aircraft measurements are shown to demonstrate practical applicability of the techniques.
\end{abstract}

\section{INTRODUCTION}

A TMOSPHERIC ice particles are dominant scatterers in cirrus clouds, thunderstorms, and winter precipitation. More than $30 \%$ of the atmosphere is covered with cirrus clouds, which play a significant role in modulating the earth's radiation budget through scattering and emission. Also, the ice mass content of a thunderstorm can be a significant portion of the total storm precipitation. On the other hand, winter storms also contain a mixture of ice crystal shapes (plates, needles, and aggregates of these) with varying bulk densities.

Complex shape extensions of ice crystals [1] such as in the case of rosettes, bullets, and stellar crystals are too small to be resolved using microwave backscatter observations in the frequency range between $S$ and $K$ bands. However, most of the ice crystals can be classified into two major categories such as plates (oblate spheroid) and needles (prolate spheroid). Hence, identification of ice crystal shapes as plates or needles through radar remote sensing may be the primary task. The

Manuscript received July 20, 1993. This work was supported under FAA Contract DTFA01-90-Z-02005. The work of J. Vivekanandan and V. N. Bringi was supported by the National Science Foundation under Grants ATM8915141 and ATM-9214864.

J. Vivekanandan is with the National Center for Atmospheric Research, Boulder, CO 80307.

V. N. Bringi is with the Department of Electrical Engineering, Colorado State University, Ft. Collins, CO.

M. Hagen and P. Meischner are with the Institut fur Physik d. Atmosphäre, DLR, Oberpfaffenhofen, Germany.

IEEE Log Number 9213506. finer details regarding shape are assumed to be of secondary importance, and complicated structures (e.g., dendritic extensions) might be handled by specifying an average dielectric constant for the corresponding volume fraction of ice. It is well known that polarimetric radar observables are sensitive to average size, shape, and bulk density of scatterers. Thus, polarimetric radar may potentially serve as a valuable tool in estimating bulk macrophysical variables such as ice water content (IWC) in clouds.

There have been a number of studies [2]-[6] to estimate IWC using radar reflectivity alone. These studies approximate nonspherical ice scatterers in terms of spherical equivalent diameter or use a specified mass-size relationship. The in situ aircraft observed particle size spectra are used to get an estimate of the variability in reflectivity $(Z)$ and IWC relationship (Z-IWC) due to the bulk density of hydrometeors. However, $Z$-IWC relationships do not show the dependence on bulk density of scatterers explicitly. At microwave frequencies, the nonspherical ice particles scatter as dipoles and backscatter amplitude is related to equivalent volume. But in reality, the ice scatterers are not only nonspherical, but are also of varying bulk density, and these conditions lead to large variabilities in a $Z$-IWC relationship. Mitchell et al. [7] report a comprehensive mass-size relationship for various habits of ice particles. Thus, any improvement in existing $Z$-IWC relationships should include explicitly both shape and bulk density of scatterers.

Differential propagation characteristics through media consisting of nonspherical scatterers such as ice crystals can be quantified in terms of the differential propagation phase since attenuation due to absorption and scattering are small. The differential phase rotates the intrinsic wavevector, resulting in depolarization of the incident wave. Depolarization causes crosstalk and attenuation in polarization diversity communication systems [8]. A number of relationships exist for rain media and dust storms to estimate differential phase and attenuation. Differential phase of $0.3^{\circ} \cdot \mathrm{km}^{-1}$ at $S$ band and $\approx 1^{\circ} \cdot \mathrm{km}^{-1}$ at $K u$ band are observed in moderate to heavy snow [9]. Electromagnetic depolarization in satellite-to-ground communication for media consisting of ice particles is well documented [11].

Polarimetric radar is a useful tool for remote sensing of media consisting of nonspherical scatterers. It is essential to characterize clouds containing ice crystals by more than one radar observable other than traditional reflectivity, such as differential propagation phase $\left(K_{D P}\right)$, linear depolarization ratio (LDR), and/or differential reflectivity $\left(Z_{D R}\right)$. The forward problem, which describes the sensitivity of various multipa- 
rameter radar observables with respect to the microphysics of ice scatterers, is studied here. Thorough understanding of the forward problem is the key to improving subsequent inverse scattering techniques of precipitation retrieval.

This paper is organized as follows. Section II describes the single scattering characteristics of ice crystals based on Rayleigh scattering and discrete dipole approximation techniques as a function of size, bulk density, and shape. Next, Section III outlines the multiparameter radar model observables which are derived by averaging single scattering characteristics over a spectrum of size, shape, and composition of ice scatterers. The relationship between IWC and radar observables are modeled for an ensemble of scatterers of varying shape, bulk density, and size distribution in Section IV. As a demonstration of potential applicability of the model described above, analysis of radar and in situ aircraft observations are presented in Section V. Finally, the paper ends with a summary and discussion of the results.

\section{SCATtERING Characteristics OF Single ICE CRystals}

Radar reflectivity due to an ensemble of scatterers in the radar resolution volume is proportional to the incoherent sum of the power backscattered from individual scatterers. Electromagnetic propagation effects through a precipitation medium can be approximated by first-order multiple scattering theory. Radar observables such as reflectivity, differential propagation phase, and differential reflectivity are all derived parameters from the principal forward and backward scattering cross sections weighted by particle size distribution (number concentration). Hence, it is essential to study principal scattering amplitudes of ice crystals as functions of size, shape, and bulk density.

The majority of ice scatterer sizes range between 0.1 and $8 \mathrm{~mm}$ equivalent spherical diameter $\left(D_{\text {eq }}\right)$ and dielectric constant $\left(\epsilon_{r}\right) \approx 3.18$, with a negligible imaginary part for particles of $0.92 \mathrm{~g} \cdot \mathrm{cm}^{-3}$. In the case of ice particles which are of lower bulk density (mixture of ice and air), the dielectric constants are even smaller than 3.18 , and mixing formulas can be used to compute the corresponding dielectric constants of particles with air and ice mixture [10]. In the frequency region between $S$ and $K$ band (3-35 GHz), the detailed structure of ice crystal shapes is less important if the sizes of the scatterers are small compared to the wavelength, as mentioned in the previous section. Ice crystals can be broadly classified either as plate-shaped and needle-shaped. The plate and needle shapes can be further approximated as oblate spheroid and prolate spheroid shapes, respectively, to make use of Rayleigh scattering techniques. Rigorous computations for column and needle-like particles are presented in [13] using the discrete dipole approximation method [14] for random orientation in the horizontal plane. Here, only scattering characteristics of plate-like crystals are discussed.

Consider an oblate spheroidal model for ice crystals of the plate type. The axis ratio $(r)$ is varied from 0.1 to 0.95 while the density $(\rho)$ is varied from 0.01 to $0.92 \mathrm{~g} \cdot \mathrm{cm}^{-3}$. The orientation is with the minor (symmetry) axis aligned along the vertical direction, also referred to as $H$ orientation. Let $D_{\text {eq }}$

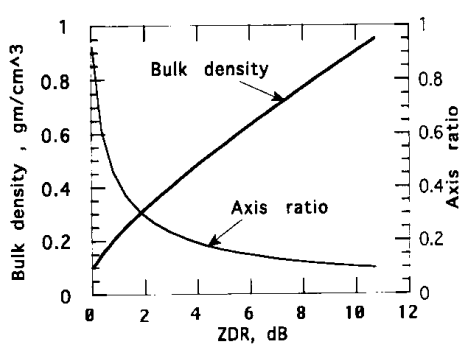

Fig. 1. $Z_{D R}$ versus axis ratio and bulk density for assumed microphysical model. See the text for details.

be the equivolumic spherical diameter. Using a numerically derived scattering amplitude, a two-dimensional curve fitting procedure was used to arrive at the following equation for the backscatter amplitude $S_{h h}$ :

$$
S_{h h}=0.063 k^{3} \rho^{0.97} D_{\mathrm{eq}}^{3}
$$

where $k$ is the wavenumber ( $=2 \pi / \lambda, \lambda$ being the wavelength). The backscatter cross section at horizontal $(h)$ polarization is given as

$$
\sigma_{B, h h}=\frac{4 \pi}{k^{2}}\left|S_{h h}\right|^{2}
$$

It should be noted that the units of $\sigma_{B, h h}$ depend on the units of the particular wavelength via the wavenumber parameter. The dependence on shape can be studied using differential backscatter and forward scatter parameters, such as differential reflectivity and the differential propagation phase. The differential reflectivity of single particles, termed $\sigma_{D R}$, is the ratio of the horizontal to vertical scattering cross section in decibels. $\sigma_{D R}$ is computed for various shape and bulk density parameters of a number of scatterers. Using a two-dimensional curve fitting, it can be shown as

$$
\begin{aligned}
& \sigma_{D R}=-11 \rho^{0.95} \log (r) \quad \sigma_{D R} \leq 5 \mathrm{~dB} \\
& \sigma_{D R}=-9.8 \rho^{0.98} \log (r) \quad 5 \mathrm{~dB} \geq \sigma_{D R} \leq 9 \mathrm{~dB} .
\end{aligned}
$$

Note that

$$
\sigma_{D R}=10 \log \frac{\left|S_{h h}\right|^{2}}{\left|S_{v v}\right|^{2}} \mathrm{~dB}
$$

Generally, as pristine ice crystals transform to aggregates, the shape becomes closer to spherical and the bulk density reduces. An ad hoc relationship such as $\rho \cdot r=0.092$ is assumed to describe such a variation in shape and bulk density. Fig. 1 shows a plot of $\rho$ and $r$ versus $\sigma_{D R}$. It is usual practice to denote differential reflectivity of a single size particle and ensemble of particles as $\sigma_{D R}$ and $Z_{D R}$, 
respectively. However, radar model computations which are discussed in this paper do not assume any dependence of shape on size of the scatterer. Thus, the notations $\sigma_{D R}$ and $Z_{D R}$ are interchangeable.

Similarly, it can be shown that the real part of the scattering amplitude difference between horizontal and vertical polarization, which is linearly related to differential propagation phase $\left(K_{D P}\right)$ for an individual scatterer, depends on size, axis ratio, and bulk density as

$$
\operatorname{Re}\left[f_{h h}-f_{v v}\right]=0.6288 k^{3}(1-r)^{1.2} D_{\mathrm{eq}}^{3} \rho^{0.967} .
$$

From the above model, it is clear that the backscatter cross section depends approximately on $\rho^{2}$, whereas both $\sigma_{D R}$ and $\operatorname{Re}\left(f_{h h}-f_{v v}\right)$ depend on $\rho$. At first approximation, $\sigma_{D R}$ is not as sensitive to axis ratio $(r)$ because of logarithmic dependence, whereas $\operatorname{Re}\left(f_{h h}-f_{v v}\right)$ is sensitive to axis ratio via $(1-r)$ dependence. As expected, the backscatter cross section is independent of axis ratio, but is strongly weighted by $D_{\text {eq }}^{6}$.

We have performed further computations using a more realistic disk model for plate-type ice crystals, rather than the oblate spheroidal model. Thin disks of $D_{\mathrm{eq}}=1.5 \mathrm{~mm}$ and thickness-to-diameter ratio of 0.025 were considered using the scattering method based on the discrete dipole approximation [14]. The disks were oriented with their long dimension in the horizontal plane termed $H$ orientation. Fig. 2(a) shows reflectivity $\left(Z_{h h}\right.$ assuming one particle per $\left.\mathrm{m}^{3}\right)$ defined as $\left(\lambda^{4} / \pi^{5}|K|^{2}\right) \sigma_{B, h h}$, where $K=\left(\epsilon_{r}-1\right) /\left(\epsilon_{r}+2\right), \epsilon_{r}$ is the dielectric constant. The wavelength is $5.5 \mathrm{~cm}$ ( $C$ band). Note that the approximate $\rho^{2}$ dependence is in good agreement with (1) and (2). Fig. 2(b) shows $\sigma_{D R}$ versus $\rho$, which is again in excellent agreement with (3) and (4). Fig. 2(c) shows $Z_{h h}$ (assuming particle concentration of one particle per $\mathrm{m}^{3}$ ) versus $D_{\text {eq }}$ where $\rho=0.2 \mathrm{~g} \cdot \mathrm{cm}^{-3}$ and thickness-to-diameter $=0.025$.

\section{MULTIPARAMETER RADAR MODEL}

The advent of pulse-to-pulse switching between horizontal $(H)$ and vertical $(V)$ polarization for transmit states and reception of the associated co- and cross-polarized returned signals has augmented the accuracy to which the precipitation medium can be characterized [12]. The multiparameter radar observables considered here are $Z_{h h}, Z_{D R}$, and $K_{D P}$. The reflectivity $\left(Z_{h h}\right)$ is related to the horizontally polarized power backscattered from a horizontally polarized transmitted wave (i.e., copolar return). The differential reflectivity $\left(Z_{D R}\right)$ is the ratio of the horizontal power received from a horizontal transmitted wave $(h h)$ to the vertical power received from a vertical wave $(v v)$. The differential propagation phase constant $\left(K_{D P}\right)$ is the difference in phase per kilometer between the two polarization states experienced by the forward traveling wave. $Z_{h h}$ and $Z_{D R}$ are expressed in logarithmic units (decibels), while $K_{D P}$ is expressed in terms of degrees of phase per kilometer.

Multiparameter radar modeling of rain, hail, graupel, and melting hydrometeors are well documented in [15]-[17]. In the case of rain media, there is a well-defined size-shape

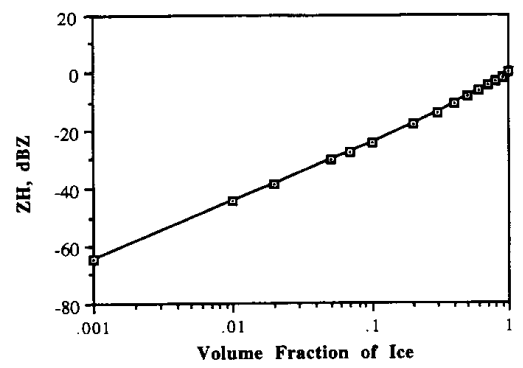

(a)

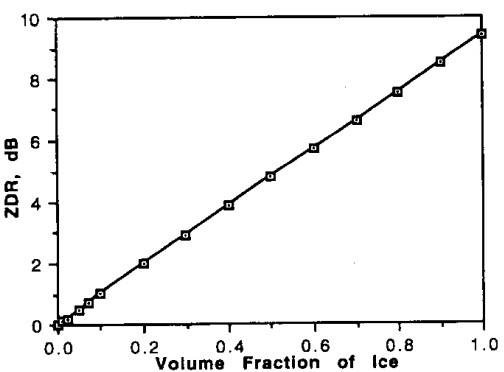

(b)

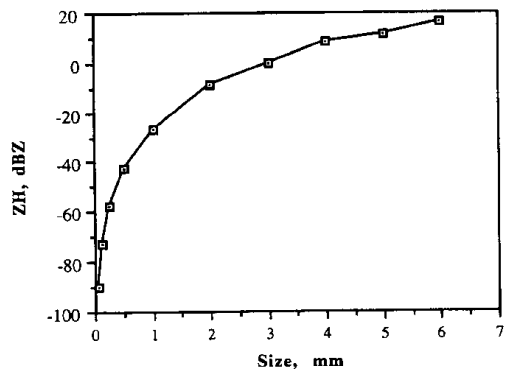

(c)

Fig 2 (a) Calculated horizontal reflectivity in dependence of volume fraction of ice for flat disks of $1.5 \mathrm{~mm}$ diameter and a thickness-to-diameter ratio of 0.025 . Particle concentration is 1 per $\mathrm{m}^{-3}$. (b) Calculated $Z_{D R}$ values for disks in (a). (c) Calculated horizontal reflectivity for disks of thickness-to-diameter ratio 0.025 as a function of particle size. Bulk density is assumed as $0.2 \mathrm{~g} \cdot \mathrm{cm}^{-3}$

relationship [18] and the bulk density is $1 \mathrm{~g} \cdot \mathrm{cm}^{-3}$. Radar measurements of snowstorms and cirrus clouds are commonly used to retrieve ice water content (IWC). But due to a large variation in bulk density and particle size distribution, the traditional reflectivity and ice water content relation varies by more than two orders of magnitude [5].

For model computation, the particle size spectrum is modeled using a gamma size distribution [19] as

$$
N(D)=N_{0} D^{m} \exp (-\Lambda D)
$$

where $\Lambda$ is the slope of the spectrum and $D$ is the equivolume particle diameter of the ice crystal. The particle axis ratio 
is varied between 0.1 and 0.95 and the bulk density of the scatterers is varied between 0.1 and $0.92 \mathrm{~g} \cdot \mathrm{cm}^{-3}$.

Multiparameter radar observables such as $Z_{h h}, Z_{v v}$, and $Z_{D R}$ for an ensemble of particles are as follows:

$$
\begin{aligned}
Z_{h h}= & \frac{4 \lambda^{4}}{(0.92) \pi^{4} k^{2}} \int_{D_{\min }}^{D_{\max }} \\
& \cdot\left|S_{h h}(D, r)\right|^{2} N(D) d D \quad\left[\mathrm{~mm}^{6} \cdot \mathrm{m}^{-3}\right] \\
Z_{v v}= & \frac{4 \lambda^{4}}{(0.92) \pi^{4} k^{2}} \int_{D_{\min }}^{D_{\max }} \\
& \cdot\left|S_{v v}(D, r)\right|^{2} N(D) d D \quad\left[\mathrm{~mm}^{6} \cdot \mathrm{m}^{-3}\right] \\
Z_{D R}= & 10 \log \left(\frac{Z_{h h}}{Z_{v v}}\right) \quad[\mathrm{dB}]
\end{aligned}
$$

where $S_{i j} i, j=v, h$ are the elements of the backscattering matrix.

The scattering process in a precipitation medium can be approximated by first-order multiple scattering. Under such conditions, the backscattered signals received by radar have both the range cumulative forward scattering and gate-bygate backscattering characteristics of the ensemble of scatterers in the medium. Range cumulative forward scattering characteristics of an ensemble of scatterers can also be recovered from pulse Doppler backscattered signals [20]. The specific differential propagation phase constant $\left(K_{D P}\right)$ is defined as

$$
\begin{aligned}
K_{D P}= & \frac{180}{\pi} \frac{2 \pi}{k^{2}} \int_{D_{\min }}^{D_{\max }} \operatorname{Re}\left(f_{h h}(D, r)\right. \\
& \left.-f_{v v}(D, r)\right) N(D) d D \quad\left[{ }^{\circ} / \mathrm{km}\right],
\end{aligned}
$$

and the two-way differential propagation phase $\phi_{D P}$ between two range locations $R_{1}$ and $R_{2}$ is defined as

$$
\phi_{D P}=2 \int_{R_{1}}^{R_{2}} K_{D P} d R .
$$

We refer to [21] for a more extensive treatment of propagation effects in rainfall at microwave frequencies. The attenuation in microwave frequencies is not considered as it is negligible for ice crystals.

\section{RELATIONSHIP BETWEEN MICROPHYSICAL PARAMETERS AND THE RADAR MODEL}

Scattering characteristics of individual scatterers depend on a number of parameters (size, shape, and bulk density), as shown in Section II. Multiparameter radar observations are the reflectivity-weighted average over the spectrum of size, shape, and bulk densities. Thus, using individual particlescattering characteristics, multiparameter radar observables can be modeled to retrieve the average microphysical parameters of the scatterers in the radar resolution volume. In this section, a number of relationships between multiparameter radar observations and average microphysical parameters are derived using model computations.

A microphysically important quantity is the ice water content (IWC), defined as

$$
\mathrm{IWC}=\frac{\pi}{6} \rho \int D_{\mathrm{eq}}^{3} N\left(D_{\mathrm{eq}}\right) d D_{\mathrm{eq}} \mathrm{g} \cdot \mathrm{m}^{-3}
$$

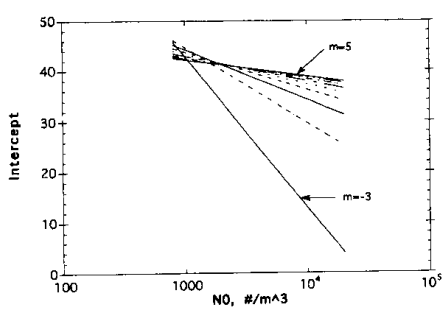

(a)

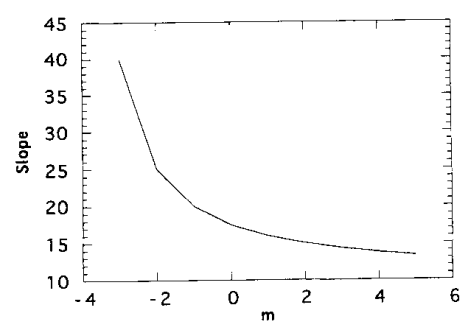

(b)

Fig. 3. Natural variations in slope and intercept of the $Z_{h h}$-IWC relationship. (a) Intercept versus $N_{0}$ for various values of $m$. (b) Slope versus $m$.

In cloud physics literature, it is conventional to estimate IWC from measurements of radar reflectivity (generally at horizontal polarization) $Z_{h h}$; however, there is a wide range of IWC for a given value of $Z_{h h}$ due to natural fluctuations in the parameters of the gamma distribution. In model computations, $N_{0}$ is varied between 800 and $18000 \mathrm{~m}^{-3} \cdot \mathrm{mm}^{-1-\mu}$, while $\mu$ is varied between -3 and 5 . The minimum and maximum $D_{\text {eq }}$ are 0.1 and $8 \mathrm{~mm}$, respectively. A general relationship between reflectivity and IWC for gamma size distribution can be obtained [19] as

$$
\begin{aligned}
Z_{h h}= & 0.242 N_{0}^{-3 /(4+m)} \Gamma(7+m) \rho^{(0.736+0.934 m) /(4+m)} \\
& \cdot\left[\frac{600}{\pi \Gamma(4+m)}\right]^{(7+m) /(4+m)} \\
& \cdot(\mathrm{IWC})^{(7+m) /(4+m)} \mathrm{mm}^{6} \cdot \mathrm{m}^{-3}
\end{aligned}
$$

In the above equation, $Z_{h h}$ is in $\mathrm{mm}^{6} \cdot \mathrm{m}^{-3}$ and IWC is in $\mathrm{g} \cdot \mathrm{m}^{-3}$. It is a standard practice to express the $Z_{h h}$ IWC relationship where $Z_{h h}$ is in $\mathrm{dBZ}$ units as

$$
Z_{h h}=\alpha+\beta \log (\text { IWC }) \mathrm{dBZ} .
$$

Comparing the above two equations, slope parameter $\beta$ depends only on $m$ and the intercept parameter $\alpha$ depends on $N_{0}, m$, and $\rho$. Fig. 3(a) shows variability in the intercept as a function of $N_{0}$ for various values of $m$; here, $\rho$ is assumed as unity. Similarly, Fig. 3(b) shows possible values of the slope parameter of the above equation. It is interesting to note that the range of values exhibited by slope and intercept parameters over natural variations of gamma size distribution parameters is in good agreement with variations exhibited in $Z_{h h}$-IWC relationships [5], [4], [3]. Under some meteorological conditions, there may be correlations among 
$N_{0}, m$, and $\rho$ that would reduce the dispersion in the $Z_{h h}-$ IWC relationship. $Z_{h h}$ is a weak function of average particle shape. The excursions in slope and intercept with regard to size distribution can also be deduced by comparing the shape of distribution, i.e., $m$ parameter. For example, in a gamma distribution with $m=4$, there is an abundance of large size particles compared to small size particles. Hence, for a given reflectivity, IWC might be smaller, and hence the intercept values are higher compared to those of negative values of $m$. Thus, by considering various microphysical variabilities in ice crystal size distribution and bulk density, the dispersion in $Z_{h h}$ versus IWC can be explained.

In our model computations, we assume that all particles have the same axis ratio and density, and that they are all oriented with a symmetry axis along the vertical $(v)$ direction or $H$ orientation. Admittedly, these assumptions are overly restrictive and idealized, but they do offer an initial approximation of the more complicated situation of a variety of shapes (axis ratios), densities, and fall modes. Using the gamma distribution, it is straightforward to show that $Z_{D R}$ is again given by (see also Fig. 1)

$$
\begin{aligned}
& Z_{D R}=-11 \rho^{0.95} \log (r) \quad Z_{D R} \leq 5 \mathrm{~dB} \\
& Z_{D R}=-9.8 \rho^{0.98} \log (r) \quad 5 \geq Z_{D R} \leq 9 \mathrm{~dB}
\end{aligned}
$$

As shown in the above relation, $Z_{D R}$ is nearly proportional to and sensitive to even small changes in $\rho$. On the other hand, relatively small changes in shape are not likely to alter $Z_{D R}$ values dramatically due to their logarithmic dependence. Thus, as a first-order approximation, $Z_{D R}$ can be used to retrieve bulk density assuming an average shape of the ice crystals. It should be noted, however, that $Z_{D R}$ as a ratio parameter does not depend on IWC [27].

The difference in scattering amplitude $\operatorname{Re}\left(f_{h h}-f_{v v}\right)$ is sensitive to all of the microphysical variables (axis ratio, bulk density, and size), as shown in (6). It is interesting to note that difference amplitude is a linear function of scatterer mass. $K_{D P}$ is derived from the scattering amplitude difference by integrating over the size distribution, as shown in Section III. The relation between $K_{D P}$ and IWC is independent of size distribution, and depends only on the average shape of the scatterer as

$$
K_{D P}=\frac{47.4}{\lambda}(1-r)^{1.2} \rho^{-0.033} \mathrm{IWC} \quad\left[{ }^{\circ} \cdot \mathrm{km}^{-1}\right]
$$

where $\lambda$ is the wavelength in millimeters. $K_{D P}$ is a weak function of $\rho$, as shown above. Thus, $K_{D P}$ is linearly related to IWC, in sharp contrast to $Z_{h h}$.

To further illustrate the properties of $K_{D P}$, consider a particle size distribution composed of oblate, oriented ice crystals mixed with "effectively" spherical-shaped snowflakes (aggregates of crystals). It is clear that the "effective" spherical snowflakes (which may be larger in size) do not contribute to KDP since $r \approx 1$. Thus, $K_{D P}$ is only sensitive to those particles that are oblate and oriented, i.e., those particles for which $\operatorname{Re}\left(f_{h h}-f_{v v}\right)$ is nonzero. However, $Z_{h h}$ is strongly weighted by the size and density of the largest particles, whereas $Z_{D R}$ depends on the reflectivity-weighted mean shape, orientation, and density of the larger particles. In the case referred to above,

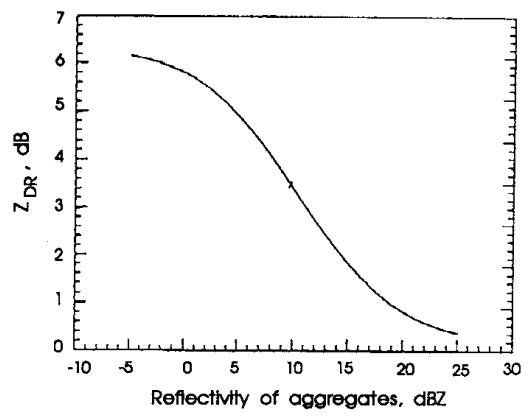

Fig. 4. Differential reflectivity of mixed-phase precipitation comprising plates and aggregates versus aggregate reflectivity. Reflectivity of plates is kept at $10 \mathrm{dBZ}$.

$Z_{h h}$ would be dominated by contribution from the snowflakes, while $Z_{D R} \approx 0 \mathrm{~dB}$ due to their "effective" sphericity. This explanation is very similar to that proposed and modeled by [22] for raindrops and hailstones; simply replace raindrops for ice crystals and hailstones for snowflakes, and the resulting interpretation for $Z_{h h}, K_{D P}$, and $Z_{D R}$ are identical.

Fig. 4 shows a plot of $Z_{D R}$ versus $Z_{h h}$ for the case of oriented, oblate ice spheroids $(r=0.2)$ mixed with tumbling aggregates $\left(r=0.8, \rho=0.2 \mathrm{~g} \cdot \mathrm{m}^{-3}\right)$ using the Mueller model described in [23]. The $Z_{h h}$ of the ice spheroids (representing pristine ice crystals) is kept constant at $10 \mathrm{dBZ}$, while the aggregate $Z_{h h}$ is varied between -5 and $25 \mathrm{dBZ}$. Note the decrease in $Z_{D R}$ from a high value of $6 \mathrm{~dB}$ when ice crystals dominate the $Z_{h h}$ to a low of near $0 \mathrm{~dB}$ when aggregates (which are "effectively" spherical) dominate the reflectivity. These calculations assume exponential size distributions, with minimum and maximum diameters of the ice crystals and aggregates being $1-5$ and $2-20 \mathrm{~mm}$, respectively. The $K_{D P}$ of the ice crystals is $0.03^{\circ} \cdot \mathrm{km}^{-1}$ (for $Z_{h h}^{\text {ice }}=10 \mathrm{dBZ}$ ); it scales with the $N_{0}$ parameter of the exponential distribution. For example, if $Z_{h h}^{\text {ice }}=20 \mathrm{dBZ}$, then $K_{D P}=0.3^{\circ} \cdot \mathrm{km}^{-1}$. The two-way $\phi_{D P}$ for a typical path length of $20 \mathrm{~km}$ will be $12^{\circ}$, which is measurable at $\lambda=10 \mathrm{~cm}$ since the standard error of $\phi_{D P}$ measurements is typically a few degrees [24].

\section{OBSERVATIONS}

The general multiparameter model computations shown in the previous sections can be applied to interpretation of multiparameter radar and aircraft observations of a winter storm. We report on observations in winter storms made with the $C$-band dual-polarized (Poldirad) radar operated by the German Aerospace Research Establishment (DLR) located in Oberpfaffenhofen, Germany. We also report on observations in the stratiform part of a mesoscale convective complex made with $S$-band radar (CIM) operated by the NOAA/National Severe Storms Laboratory. A description of these radars is available in [12]. The Poldirad system description can be found in [25]. We first present examples of data from Poldirad and CIM, and then present a more detailed case using Poldirad, i.e., the November 27, 1987 case, where a cloud physics aircraft was used to validate the $Z_{D R}$ radar signatures. 

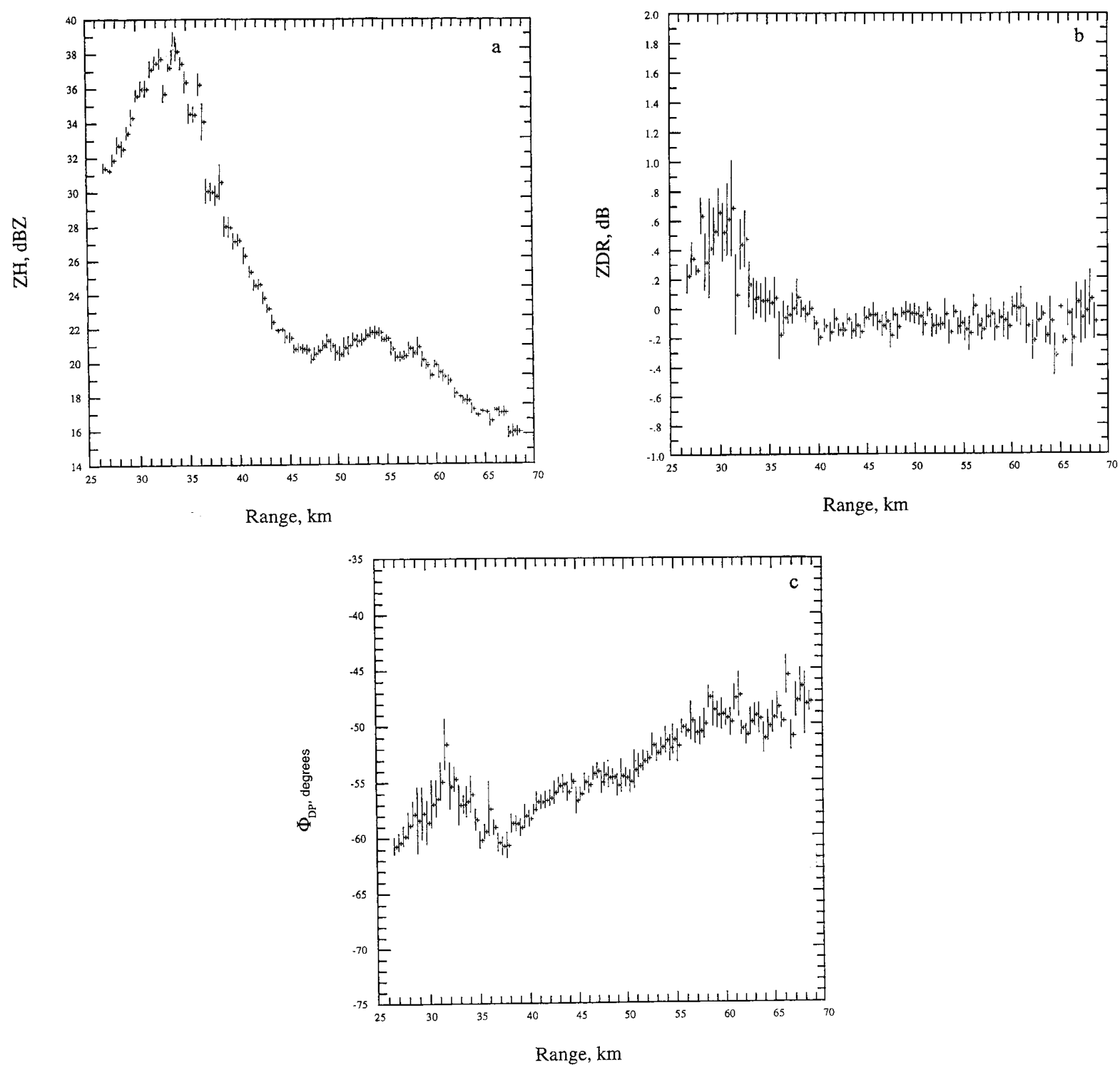

Fig. 5. The range profiles of (a) $Z_{h h}$, (b) $Z_{D R}$, and (c) $\phi_{D P}$ taken at an elevation angle of $5^{\circ}$. These data have been averaged over eight consecutive beams with the antenna fixed. The vertical bars show the standard derivations.

\section{A. Example from June 3, 1989 Using CIM}

On June 3, 1989, the CIM radar collected RHI data in the stratiform region of a mesoscale convective complex. A welldefined bright band in $Z_{h h}$ was observed near the $0^{\circ} \mathrm{C}$ level. Detailed polarimetric observations and interpretation within the bright-band region are reported in [28]. In this paper, we focus on $\phi_{D P}$ range profiles above the bright band. Fig. 5 shows the range profiles of (a) $Z_{h h}$, (b) $Z_{D R}$, and (c) $\phi_{D P}$ taken at an elevation angle of $5^{\circ}$. These data have been averaged over eight consecutive beams $(2 \mathrm{~s})$ with the antenna fixed. Vertical bars show the standard deviations. In Fig. 5(a), the bright band in $Z_{h h}$ is clearly visible at range $=35 \mathrm{~km}$ (height $=3 \mathrm{~km}$ ). The environmental $0^{\circ} \mathrm{C}$ level was at $3.4 \mathrm{~km}$ agl. Fig. 5(b) also shows a bright band in $Z_{D R}$, a feature which was first observed in [26]. Above the bright band (ranges beyond $40 \mathrm{~km}$ ), the $Z_{D R}$ is close to $0 \mathrm{~dB}$, while $Z_{h h}$ shows an overall decreasing trend and lies in the range $18-25 \mathrm{dBZ}$. The $\phi_{D P}$ range profile in Fig. 5(c) shows an increasing trend beyond a range of $40 \mathrm{~km}$. This is most likely caused by $H$ oriented ice crystals of the dendritic type. The $\phi_{D P}$ fluctuation within the bright band is due to the backscatter differential phase, as discussed in [28]. In the range interval $40-60 \mathrm{~km}$, the two-way $\phi_{D P}$ changes by $10^{\circ}$, giving the average specific differential phase $K_{D P}$ of $0.25^{\circ} \cdot \mathrm{km}^{-1}$. There are regions of higher and lower $K_{D P}$ within this range interval, e.g., values up to $0.5^{\circ} \cdot \mathrm{km}^{-1}$ in the interval $40-45 \mathrm{~km}$, and values as low as $0.1^{\circ} \cdot \mathrm{km}$ in the range $45-50 \mathrm{~km}$. These values of 


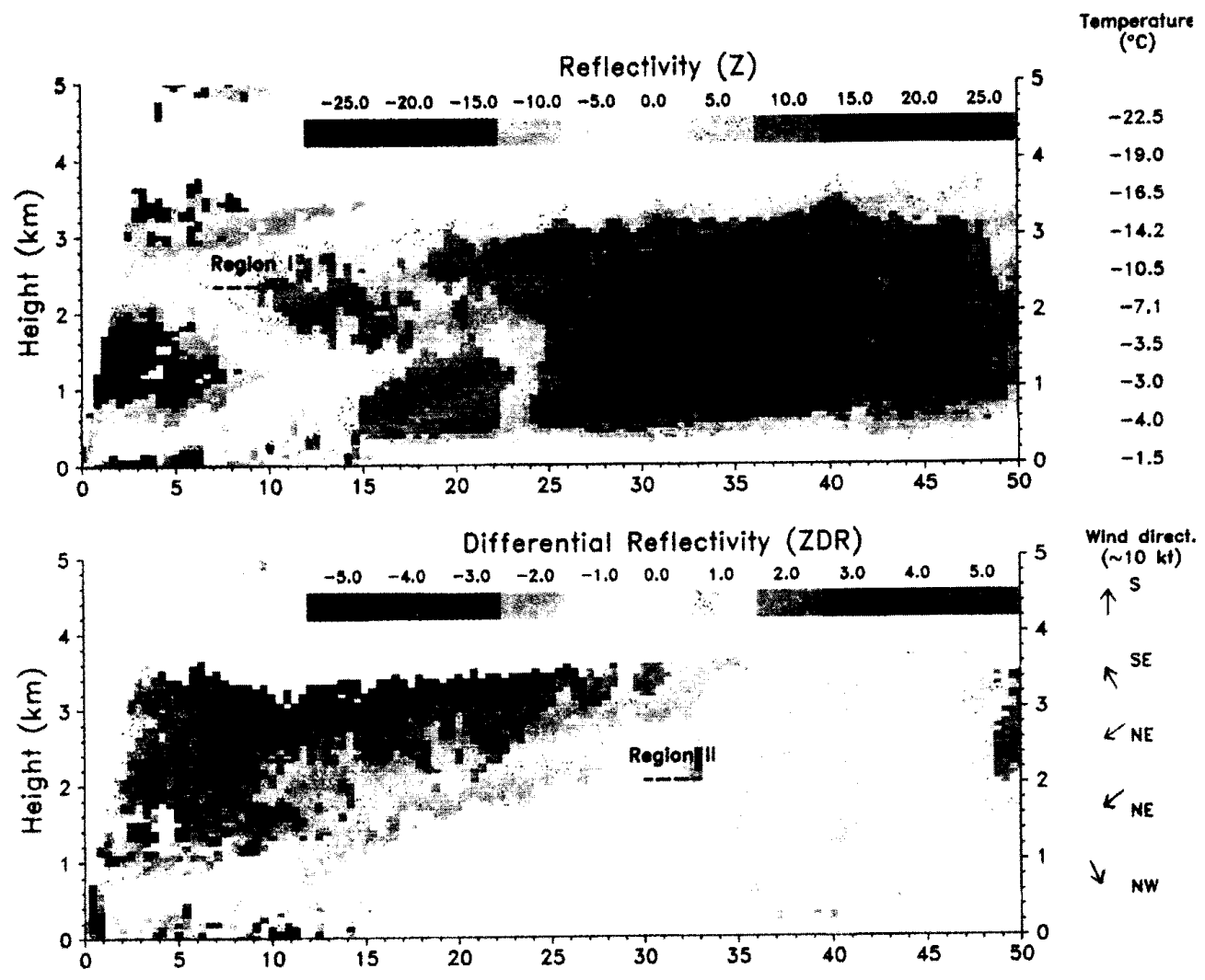

Fig. 6. RHI along the $210^{\circ}$ azimuth showing reflectivity $\left(Z_{h h_{h}}\right)$ and differential reflectivity $\left(Z_{D R}\right)$ on November 27,1987 at 1011 local time. Regions I and II indicate areas where aircraft data were analyzed.

$K_{D P}$ are in reasonable agreement with Hendry et al . [9] who derived $K_{D P}\left(\sim 0.36^{\circ} \cdot \mathrm{km}^{-1}\right)$ at $S$ band from dual circularly polarized radar observations in what they called snow. The high $K_{D P}$ values of $0.5^{\circ} \cdot \mathrm{km}^{-1}$ in the range sector $40-45 \mathrm{~km}$ is interesting (corresponding to a height interval of 3.5-3.9 $\mathrm{km}$ agl). In this region, the $Z_{h h}$ increases very rapidly from $21 \mathrm{dBZ}$ at $45 \mathrm{~km}$ to $27 \mathrm{dBZ}$ at $40 \mathrm{~km}$, while $Z_{D R}$ is very near $0 \mathrm{~dB}$. Fig. 4 models this behavior. The high $K_{D P}$ might have been caused by $H$-oriented ice crystals, possibly dendrites.

\section{B. Case of November 27, 1987 Using Poldirad}

On this day, an upslope situation developed at the northern Alpine area caused by a low-pressure system to the south of the Alps. Moist Mediterranean air was forced around the east of the Alps, and low-level winds from the north maintained the upslope conditions during the observation period. First layer clouds initially formed southwest of Oberpfaffenhofen. During the forenoon, the system developed into widespread snowfall. Additional moisture was provided by southerly flow across the Alps above $4 \mathrm{~km}$ MSL. Radar measurements show increasing precipitation rates toward the Alps. The NCAR King Air penetrated the winter storm at four flight levels between 1450 and $2450 \mathrm{~m}$ above ground along the $210^{\circ}$ radial with respect to the radar. The excellent navigation of this plane ensured compatibility of both the radar and in situ measurements. PMS data-imaged particles are classified by manual inspection in different categories (dendrites, aggregates, and graupel), and their relative number concentrations are estimated.

Fig. 6 shows a two-panel RHI section of reflectivity $Z_{h h}$ (also termed $Z$ ) in the top panel and $Z_{D R}$ in the bottom panel. The aircraft was frequently detected by radar, as can be seen in Fig. 6, at $32.5 \mathrm{~km}$ range, $2.4 \mathrm{~km}$ above ground level. Two regions were selected to show the radar parameters of different kinds of ice particles. At ranges between 7 and 11 $\mathrm{km}$ (region $\mathrm{I}$ ), low reflectivity $(-5-10 \mathrm{dBZ})$ is accompanied by relatively high values of $Z_{D R}(2-4 \mathrm{~dB})$. A scattergram of the observed radar parameters in this region $(200 \mathrm{~m}$ above and below the flight path) is shown in Fig. 7(a). The estimated size distributions from the aircraft data for dendrites, aggregates, and graupel are shown in Fig. 7(b). Due to their shape, dendrites will have higher $Z_{D R}$ than graupel and aggregates. However, $Z_{D R}$ values will be biased toward a lower value if the dendrites coexist with graupel and aggregates. In this region, concentration of dendrites is dominant, and this agrees well with observed polarization signature. Region II is selected where reflectivity is relatively high (around $15 \mathrm{dBZ}$ ) and $Z_{D R}$ is about zero [Fig. 7(c)]. Fig. 7 (d) shows the particle concentration for Region II (between 30 and $33 \mathrm{~km}$ range). Aggregates constitute the major type of scatterers, and occurring dendrites are essentially rimed. 


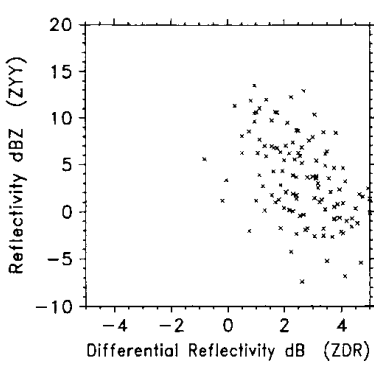

(a)

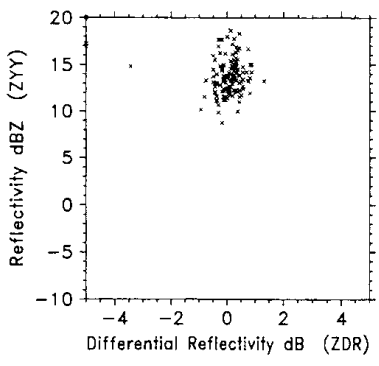

(c)

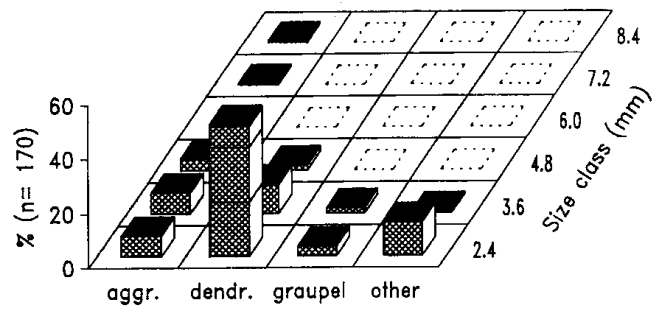

(b)

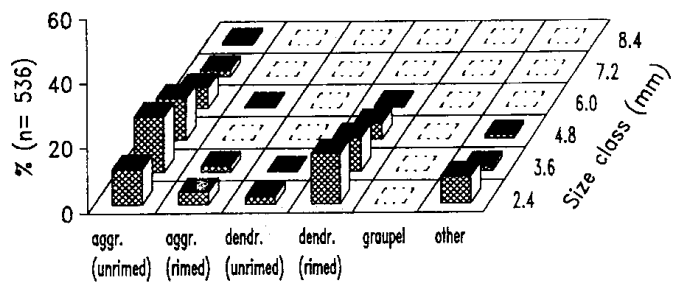

(d)

Fig. 7. Scattergram of radar parameters $Z_{h h}$ versus $Z_{D R}$ and particle classification results obtained for Region I (a) and (b), and for Region II (c) and (d).

Thus, detailed analysis of PMS images revealed that the regions with low reflectivity and high $Z_{D R}$ correspond to relatively higher number concentrations of dendrites $(\leq 3.6$ $\mathrm{mm}$ ). Similarly, the regions of aggregates (up to $6-7 \mathrm{~mm}$ ) correspond to high reflectivity and low $Z_{D R}$ values. These observations are consistent with earlier results [27]. Thus, the particle classification results show general agreement with the expected polarization signatures. However, it is difficult to estimate IWC from aircraft measurements since there are no suitable instruments for direct measurement. A method which combines radar and aircraft measurements results in considerable error due to large differences in radar and aircraft sampling volumes.

For a medium consisting of a mixture of particles of varying bulk densities and sizes, it is especially difficult to estimate the IWC. In practice, most of winter storm observations contain a mixture of ice scatterers such as dendrites, rimed dendrites, and aggregates. The modeling results presented in previous sections are valid, but only if the scatterers are of the same shape and bulk density of varying sizes in a given radar resolution volume. To handle this type of complex problem within a limited modeling approach, an approximate method is proposed and described below.

In general, as the dendritic ice crystals transform to aggregates, bulk density reduces and the shape becomes closer to spherical. Thus, the polarimetric response becomes less pronounced with the onset of aggregation. Also, there is a change in size and bulk density which leads to variations in reflectivity and IWC. Pristine plate-like ice crystals will have higher bulk density and a small axis ratio. It may be assumed that a pristine plate with a bulk density of $0.92 \mathrm{~g} \cdot \mathrm{cm}^{-3}$. has an axis ratio of 0.1 , and the fully aggregated ice crystal will have an axis ratio of 0.92 and low bulk density of $0.1 \mathrm{~g}$. $\mathrm{cm}^{-3}$. Then, the transformation process between pristine plate and aggregate can be approximated, using the axis ratio $(r)$ and bulk density $(\rho)$ as

$$
\rho \cdot r=0.092 \text {. }
$$

Fig. 1 shows the variation of $\rho$ and $r$ versus corresponding $Z_{D R}$. Thus, the value of $\rho$ or $r$ can be inferred from $Z_{D R}$. Then, either $K_{D P}$ observations can be directly used to infer IWC or, assuming the values of $N_{0}$ and $m$ parameters of size, distribution IWC can be obtained from the $Z_{h h}$-IWC expression. Detailed cloud model results in combination with rigorous radar modeling studies can be used to verify the precipitation retrieval techniques outlined above.

\section{CONCLUSIONS}

Polarimetric radar techniques for ice scatterers were studied using the nonspherical nature and orientation of scatterers. It is shown that measurements of $Z_{h h}, Z_{D R}$, and $K_{D P}$ can qualitatively be used to identify oriented ice crystals and the transformation of crystals into aggregates. The data reported are consistent with simple models of plate-type ice crystals, e.g., stellar or dendritic crystals which orient themselves by their long dimension in the horizontal plane. Even though polarimetric signature depends on a number of microphysical characteristics (size, shape, and bulk density), it may be possible to identify the individual microphysical characteristics. For example, $Z_{D R}$ depends both on bulk density and axis ratio. 
However, only the logarithm of the axis ratio $(r)$ is involved in the $Z_{D R}$ relation. Thus, as a first-order approximation, it may be possible to estimate bulk density using $Z_{D R}$.

The model shows the functional relationship among $Z_{h h}, Z_{D R}$, and $K_{D P}$ with particle shape, density, and IWC. The variability in slope $\left[\beta\right.$ in (15)] of the $Z_{h h}$-IWC relation depends on the shape of the size distribution $(m)$ alone. The excursions in the intercept $[\alpha$ in (15)] and the slope of the relationship can be explained by the variabilities in size distribution parameters. It is interesting to note that a nonlinear relationship (IWC is related to the square root of $Z_{h h}$ ) between $Z_{h h}$ IWC is the cause of the dependence upon size distribution. Thus, any improvement in the $Z_{h h}$-IWC relationship might have to address the variability in size distribution parameters. For a cloud composed of ice crystals only, $K_{D P}$ is not sensitive to size distribution, and hence it may be used for IWC estimation, provided the average density and axis ratio of the ice crystals are known. The transformation of ice crystals into aggregates is accompanied by an increase in $Z_{h h}$ and a corresponding reduction in $Z_{D R}$, which is in good, qualitative agreement with simulations and measurements.

$Z_{h h}$ and $Z_{D R}$ observations in winter precipitation using the $C$-band Poldirad system are discussed. Radar observations of $Z_{h h}$ and $Z_{D R}$ are compared with in situ aircraft measurements of particle size and shape. The in situ particle data were manually classified into three particle types: dendritic ice crystals, aggregates, and graupel. It is shown that the observations can be mapped into a $Z_{h h}-Z_{D R}$ plane where particle classification between aggregates and dendrites appears feasible. A simple model is proposed based on observations in one case study for the transformation of dendrites into aggregates that can be remotely sensed using radar measurements of $Z_{h h}, Z_{D R}$, and $K_{D P}$.

Observations of $Z_{h h}, Z_{D R}$, and $K_{D P}$ in the stratiform region of a mesoscale convective complex using the $S$-band $\mathrm{CIM}$ radar are discussed. Above the $0^{\circ} \mathrm{C}$ level, the $K_{D P}$ values range from 0.2 to $0.5^{\circ} \cdot \mathrm{km}^{-1}$, which are in good agreement with previous studies at $S$ band in snow [9]. It is clearly seen that particles responsible for the $Z_{h h}$ and $Z_{D R}$ signatures are different from particles responsible for $K_{D P}$. It is concluded that, while it is difficult to quantify winter precipitation because of the wide variety of shapes, densities, fall modes, and size distributions, the additional information supplied by $Z_{D R}$ and $K_{D P}$ can be very useful in radar remote sensing of winter precipitation or precipitation existing in regions colder than $0^{\circ} \mathrm{C}$.

\section{ACKNOWLEDGMENT}

The NCAR King Air aircraft was supported by NSF and operated by the Research Aviation Facility of NCAR. The authors acknowledge NOAA's National Severe Storm Laboratory which operates the $S$-band CIM radar, and, in particular, Dr. D. S. Zrnic of NOAA, who made it possible for the second author (V. N. Bringi) to collect data with this radar. Dr. J. Hubbert of Colorado State University is thanked for developing analysis software used to generate Fig. 5 .

\section{REFERENCES}

[1] C. Magono and C. V. Lee, "Meteorological classification of natural snow crystals," J. Fac. Sci., Hokkaido Univ., ser. 7, no. 2, pp. 321-362, 1966

[2] R. S. Sekhon and R. C. Srivastava, "Snow size spectra and radar reflectivity," J. Atmos. Sci., vol. 27, pp. 299-307.

[3] A. J. Heymsfield, "Precipitation development in stratiform ice clouds: A microphysical and dynamical study," J. Atmos. Sci., vol. 34, pp. 367-381, 1977.

[4] P. H. Herzegh and P. V. Hobbs, "The mesoscale and microscale structure and organization of clouds and precipitation in midlatitude cyclones. II: Warm frontal clouds," J. Atmos. Sci., vol. 37, pp. 597-611, 1980.

[5] A. J. Heymsfield, "Relationships for deriving thunderstorm anvil ice mass for CCOPE storm water budget estimates," J. Climate Appl. Meterol., vol. 25, pp. 691-702, 1986.

[6] Y. Fujiyoshi, T. Endoh, T. Yamada, K. Tsuboki, Y. Tachibana, and G. Wakahama, "Determination of $Z-R$ relationship for snowfall using a radar and high sensitivity snow gauges," J. Appl. Meteorol., vol. 29, pp. $147-152,1990$.

[7] D. L. Mitchell, R. Zhang, and R. L. Pitter, "Mass-dimensional relationships for ice particles and the influence of riming on snowfall rates," $J$. Appl. Meteorol., vol. 29, pp. 153-163, 1990.

[8] T. S. Chu, "Effects of sandstorms on microwave propagation," Bell Syst. Tech. J., pp. 549-555, 1978.

[9] A. Hendry, G. C. McCormick, and B. L. Barge, " $K u$-band and $S$-band observations of the differential propagation constant in snow," IEEE Trans. Antennas Propagat, vol. AP-24, pp. 521-525, 1976.

[10] M. E. Turi, "Theoretical and experimental studies of microwave emission signatures of snow," IEEE Trans. Geosci. Remote Sensing, vol. 20 , pp. 51-57, 1982.

[11] D. C. Cox, H. W. Arnold, and H. H. Hoffman, "Depolarization of 19 and $28 \mathrm{GHz}$ earth-space signals by ice particles," Radio Sci., vol. 13, May-June, 1978.

[12] V. N. Bringi and A. Hendry, "Technology of polarization diversity radars for meteorology," in Radar in Meteorology, D. Atlas, Ed., AMS, 1990, ch. 19 a, pp. 153-190.

[13] K. F. Evans and J. Vivekanandan, "Multiparameter radar and microwave radiative transfer modeling of nonspherical atmospheric ice particles," IEEE Trans. Geosci. Remote Sensing, vol. 28, no. 4, pp. 423-437, 1990.

[14] G. H. Goedecke and S. G. O'Brien, "Scattering by irregular inhomogeneous particles via the digitized Green's function algorithm," Appl. Opt., vol. 27, no. 12, pp. 2431-2438, 1988

[15] V. N. Bringi, R. M. Rasmussen, and J. Vivekanandan, "Multiparameter radar measurements in Colorado convective storms. Part I: Graupel melting studies," J. Atmos. Sci., vol. 43, pp. 2545-2563, 1986.

[16] K. Aydin, T. A. Seliga, and V. N. Bringi, "Differential radar scattering properties of model hail and mixed phase hydrometeors," Radio Sci., vol. 19 , pp. $58-66,1984$.

[17] J. Vivekanandan, V. N. Bringi, and R. Raghavan, "Multiparameter radar modeling and observations of melting ice," J. Atmos. Sci., vol. 47, no. 5, pp. 549-563, 1990.

[18] H. R. Pruppacher and R. L. Pitter, "A semi-empirical determination of the shape of cloud and rain drops," J. Atmos. Sci., vol. 28, pp. 86-94, 1971.

[19] C. W. Ulbrich, "Natural variations in the analytical form of the raindrop size distribution," J. Climate Appl. Meteorol., vol. 22, pp. 1764-1755, 1983.

[20] A. J. Jameson and E. A. Mueller, "Estimation of propagation differential phase shift from sequential orthogonal linear polarization radar measurements," J. Atmos. Oceanic Tech., vol. 2, pp. 133-137, 1985.

[21] V. N. Bringi, V. Chandrasekar, N. Balakrishnan, and D. S. Zrnic, "An examination of propagation effects in rainfall on radar measurements at microwave frequencies," J. Atmos. Oceanic Tech., vol. 6, pp. 829-840, 1990.

[22] N. Balakrishnan and D. S. Zrnic, "Estimation of rain and hail rates in mixed-phase precipitation," J. Atmos. Sci., vol. 47, pp. 565-583, 1990.

[23] J. Vivekanandan, R. Raghavan, and V. N. Bringi, "Polarimetric radar modeling of mixtures of precipitation particles," in Proc. Int. Geosci. Remote Sensing Symp., 1992, pp. 1379-1382.

[24] M. Sachidananda and D. S. Zrnic, "Differential propagation phase shift and rainfall rate estimation," Radio Sci., vol. 21, p. 235-247, 1986.

[25] A. C. Schroth, M. S. Chandra, and P. F. Meischner, "A. C-band coherent polarimetric radar for propagation and cloud physics research," J. Atmos. Oceanic Tech., vol. 5, pp. 803-822, 1988.

[26] M. P. M. Hall, W. F. Goddard, and S. M. Cherry, "Identification of hydrometeors and other targets by dual-polarization radar," Radio Sci., vol. 19, pp. 132-140, 1984. 
[27] M. J. Bader, S. A. Clough, and G. P. Cox, "Aircraft and dual polarization radar observations of hydrometeors in light stratiform precipitation," $Q$. J. R. Meteorol. Soc., vol. 115, pp. 491-515, 1987.

[28] D. S. Zrnic, N. Balakrishnan, C. L. Ziegler, V. N. Bringi, and K. Aydin, "Polarimetric signatures in the stratiform region of a mesoscale convective system," accepted for publication, J. Appl. Meteor., 1992.

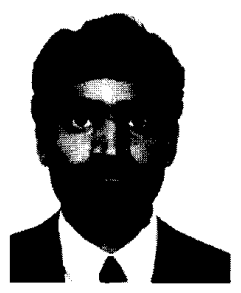

J. Vivekanandan received the B.E. degree in electronics and communications engineering from Madurai-Kamaraj University, the M.Tech. degree in microwave and radar engineering from the Indian Institute of Technology, Kharagpur, and the Ph.D. degree in electrical engineering from Colorado State University, Ft. Collins, in 1986.

At present, he is a Scientist at the National Center for Atmospheric Research, Boulder, CO. His research experience includes microwave radar and radiometer/satellite remote sensing of the atmosphere, vegetation, and soil. Recently, he has devoted considerable effort toward modeling polarimetric radar and multifrequency radiometer observations in clouds and their interpretations.

V. N. Bringi's research interests over the last 15 years have been in the areas of electromagnetic wave propagation and scattering, multiple scattering of waves, polarimetric radar techniques and applications to meteorology.

Dr. was a member of AMS Committee on Radar Meteorology. He is currently a member of the NEXRAD Technical Advisory Committee. He has received the following honors/awards: UCAR Fellowship (1974-1976), Alumni award for Graduate Student Research (1977, The Ohio State University), CSU College of Engineering's Young Faculty Professional Development Award (1983), the Halliburton Young Faculty Research Award (1984), and the Dean's Council Award (1989).

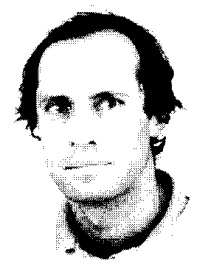

Martin Hagen received the M.S. degree in meteorology from the University of Karlsruhe, Germany, in 1986, and the Ph.D. degree

Following his master's degree he started research with polarimetric Doppler radar at the Institute of Atmospheric Physics at the Deutsche Forschungsanstalt fuer Luft-und Raumfahrt (DLR), Oberpfaffenhofen, Germany, and received the Ph.D. degree from the University of Munich in 1989. In his thesis he developed a single Doppler wind field estimation algorithm and discussed the effect of the Alps on cold fronts. His main research interest currently is in the identification of hydrometeors by polarimetric radar.

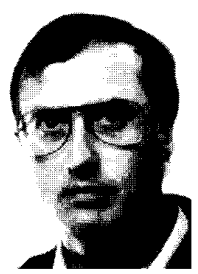

Peter F. Meischner is a physicist who completed his academic education at the Free University Berlin and the Max Planck Gesellschaft.

Since 1983, he has been head of the Cloud Physics Division at DLR, Institute of Atmospheric Physics, Oberpfaffenhofen. His research interest covers cloud physics and cloud dynamics of cloud systems and complexes. He planned and directed several field experiments as CLEOPATRA-92. Together with the DLR Institute of High Frequency Technology, he designed POLDIRAD, which has been used for cloud physics research and wave propagation studies since 1986. He has been chairman of the European COST 75 action since 1993. 